\title{
Dislocation Glide-Controlled Room-Temperature Plasticity in 6H-SiC Single Crystals
}

\author{
S. Kiani ${ }^{\mathrm{a}}$, K.W.K. Leung ${ }^{\mathrm{b}}$, V. Radmilovic ${ }^{\mathrm{c}}$, A. M. Minor ${ }^{\mathrm{d}, \mathrm{e}}$, J.-M. Yang ${ }^{\mathrm{a}}$, \\ D.H. Warner ${ }^{b^{*}}$, and S. Kodambaka ${ }^{a^{*}}$ \\ ${ }^{a}$ Department of Materials Science and Engineering, University of California Los Angeles, Los \\ Angeles, CA 90095, USA \\ ${ }^{\mathrm{b}}$ School of Civil and Environmental Engineering, Cornell University, Ithaca, NY 14853, USA \\ ${ }^{\mathrm{c}}$ Nanotechnology and Functional Materials Center, Faculty of Technology and Metallurgy, \\ University of Belgrade, Belgrade, Serbia \\ ${ }^{\mathrm{d}}$ National Center for Electron Microscopy, Lawrence Berkeley National Laboratory, Berkeley, \\ CA 94720, USA \\ ${ }^{\mathrm{e}}$ Department of Materials Science and Engineering, University of California Berkeley, Berkeley, \\ CA 94720, USA
}

\begin{abstract}
In situ transmission electron microscopy observations of uniaxial compression of sub 300 $\mathrm{nm}$ diameter, cylindrical, single-crystalline $6 \mathrm{H}-\mathrm{SiC}$ pillars oriented along $\langle 0001\rangle$ and at $45^{\circ}$ with respect to $\langle 0001\rangle$ reveal that plastic slip occurs at room-temperature on the basal $\{0001\}$ planes at stresses above 7.8 GPa. Using a combination of aberration-corrected electron microscopy, molecular dynamics simulations, and density functional theory calculations, we attribute the observed phenomenon to basal slip on the shuffle set along $\langle 1100\rangle$. By comparing the experimentally-measured yield stresses with the calculated values required for dislocation nucleation, we suggest that room-temperature plastic deformation in $6 \mathrm{H}-\mathrm{SiC}$ crystals is controlled by glide rather than nucleation of dislocations.
\end{abstract}

Keywords: Transmission Electron Microscope, Plasticity, Dislocations, Silicon carbide, Molecular Dynamics

\footnotetext{
* Corresponding authors: kodambaka@ucla.edu; dhw52@cornell.edu
} 


\section{Introduction}

The critical load bearing components of many modern structures are often constructed from metallic alloys having lower strengths than their ceramic counterparts. This design compromise is often driven by the need for component reliability, a trait that is generally superior in metallic alloys due to their ability to deform plastically at room-temperature. The availability of higher strength materials with enhanced ductility would significantly boost the performance and enable next-generation advanced technologies. This, however, is a challenge and requires fundamental understanding of the factors influencing mechanical properties of materials. In case of metals, recent investigations have helped identifying the role of crystal size and microstructure on their mechanical properties $[1,2]$. For higher strength refractory materials such as $\mathrm{SiC}$, a covalently bonded structural ceramic and a semiconductor, most of the existing literature is focused on the mechanical behavior of bulk crystals at elevated temperatures $(\mathrm{T}>$ $673 \mathrm{~K})$ [3-7] as they are expected to be brittle at room-temperature. For example, hightemperature compression and bending of bulk $4 \mathrm{H}-\mathrm{SiC}$ crystals revealed the existence of partial dislocations on the basal $\{0001\}$ planes, full dislocations out of the basal planes, and stacking faults due to $4 \mathrm{H}$ to $3 \mathrm{C}$ transformation $[8,9]$. Room-temperature measurements of indentation hardness [10-12] of bulk single-crystalline $\mathrm{SiC}$ and fracture strengths $[13,14]$ of SiC nanorods and nanowires have helped identify the role of indenter geometry, crystal size, and crystal orientation on the mechanical properties of SiC. Room-temperature plasticity, although not common, has been observed in crystalline $\beta$-SiC nanowires under tension and attributed to formation and motion of dislocations followed by transition to an amorphous state $[15,16]$. Here, we focus on understanding the room-temperature mechanical behavior of single-crystalline $6 \mathrm{H}-$ $\mathrm{SiC}$ (a polytype of $\alpha-\mathrm{SiC}$ ), a hard (> $25 \mathrm{GPa}$ ), stiff (elastic modulus > $415 \mathrm{GPa}$ ), and high 
melting point $\left(\mathrm{T}_{\mathrm{m}} \approx 3000 \mathrm{~K}\right)$ solid subjected to uniaxial compression [17-19]. We show that small (< $300 \mathrm{~nm}$ in diameter) $6 \mathrm{H}-\mathrm{SiC}$ single-crystals deform plastically at room-temperature under a compressive load, exhibiting a long sought combination of mechanical properties, high yield strength $(>7.8 \mathrm{GPa})$, and considerable ( 10\% strain) plastic flow before failure, indicative of ductility. By combining in situ transmission electron microscopy (TEM) based nanomechanical testing with aberration-corrected TEM, molecular dynamics (MD) simulations, and density functional theory (DFT) calculations, we identify the mechanism leading to plastic flow in single-crystalline $6 \mathrm{H}-\mathrm{SiC}$.

\section{Methods}

\subsection{Experimental details}

All the in situ compression experiments are conducted on cylindrical SiC pillars prepared out of $5 \times 5 \times 0.33 \mathrm{~mm} 6 \mathrm{H}-\mathrm{SiC}(0001)$ single-crystals purchased from MTI Corporation. A combination of mechanical polishing and focused ion beam (FIB) milling are used to prepare $\Phi_{0}$ and $\Phi_{45}$ pillars oriented along $\langle 0001\rangle$ and at $45^{\circ}$ with respect to $\langle 0001\rangle$, respectively.

In order to prepare $\Phi_{0}$ pillars, which are uniaxially compressed in the direction normal to the basal plane, a $1 \times 1 \times 0.33 \mathrm{~mm}$ piece is first cut out of the larger crystal with a diamond saw and mechanically thinned to sub-100 $\mu \mathrm{m}$ thickness using Multiprep auto-polisher (Allied High Tech Products, Inc.). Then, the sample is mounted on a copper half-grid using M-bond 610 adhesive (Micro-Measurements) and cured at $160{ }^{\circ} \mathrm{C}$ for 1.5 hours. This process ensures that the thinned sample is permanently bonded to the half-grid. The grid-sample is attached to a copper sample mount (from Hysitron Inc.) using CrystalbondTM 509 adhesive (Ted Pella, Inc.) and transferred to a FEI 235 dual beam FIB system for milling. (Fig. S1 in Supplementary 
Information shows a schematic of the sample assembly used for milling.) The whole assembly (sample + copper-half-grid + copper sample mount) is attached to a 45-degree stub using Pelco colloidal graphite paste (Ted Pella, Inc.) to maximize electrical conductivity and to minimize drift during FIB milling. FIB milling is carried out using $30 \mathrm{kV} \mathrm{Ga}^{+}$beams in two steps: initial coarse milling at an ion beam current of $7 \mathrm{nA}$ followed by finer milling with $30 \mathrm{pA}$ current. The final step involves a low-voltage $(6 \mathrm{kV})$ milling to minimize $\mathrm{Ga}^{+}$implantation in, and the formation of amorphous layers on, the pillars. The resulting pillar diameters $(D)$ are between 170 $\mathrm{nm}$ and $280 \mathrm{~nm}$, with aspect ratios chosen to be less than 3.5 to minimize buckling.

We follow a similar procedure to prepare $\Phi_{45}$ pillars, whose loading direction is $45^{\circ}$ with respect to $\langle 0001\rangle$. The key difference is that the $1 \times 1 \times 0.33 \mathrm{~mm}$ piece cut out from the bulk crystal is mounted on a 45-degree stub and mechanically polished using the Multiprep autopolisher to create a $45^{\circ}$-cut surface. Then the sample is remounted on one side and mechanically thinned from $0.33 \mathrm{~mm}$ thickness to sub-100 $\mu \mathrm{m}$ using the Multiprep auto-polisher. All the other pillar fabrication steps are as described in the previous section.

The copper sample mount is then loaded into a Hysitron PicoIndenter 95 with a flat 3$\mu \mathrm{m}$-wide diamond punch for in situ uniaxial compression at room-temperature inside a JEOL 3010 TEM operated at $300 \mathrm{kV}$. The applied load is gradually increased such that the displacement changes at a rate of $4 \mathrm{~nm} / \mathrm{s}$. During compression of each pillar, load $v s$. displacement data, video-rate (30 frames/s) TEM images, and selected area electron diffraction (SAED) patterns are acquired. The engineering stress and strain values are calculated from the load-displacement data using the lengths and diameters of the undeformed pillars.

\subsection{Molecular dynamics (MD) simulations and density functional theory (DFT) calculations}


The MD simulations are performed using LAMMPS package [20] with Devanathan et al.[21] potential for $\mathrm{SiC}$. This potential reliably reproduces equilibrium properties of the crystal including lattice parameters, elastic constants, cohesive energy, and point defect formation energy. In the simulation, cylindrical and defect-free $6 \mathrm{H}-\mathrm{SiC}$ single-crystals with diameter $D=$ $8.4 \mathrm{~nm}$ and a height of $24 \mathrm{~nm}$, consisting of 133,690 atoms, are oriented such that the direction of the applied load is $45^{\circ}$ with respect to $\langle 0001\rangle$. The simulation cell is first thermalized for $10 \mathrm{ps}$ at $300 \mathrm{~K}$ to release surface stresses in all directions and to initialize atomic positions and velocities. The cell temperature is controlled by a Langevin thermostat [22]. A canonical (NVT) ensemble, where $\mathrm{N}$ is the number of particles in a system of volume $\mathrm{V}$ held at an absolute temperature $\mathrm{T}$, is employed with an integration time step of 1 fs for the velocity-Verlet integration scheme [23]. Uniaxial compression is simulated by imposing deformation on the cell at a strain rate of $5.7 \times 10^{9} \mathrm{~s}^{-1}$. Stresses are computed with the virial theorem [24] and strains are determined from the changes in the crystal height. Atomeye package is used for atomistic visualization of the crystal [25].

The DFT simulation cell is a fully periodic $6 \mathrm{H}-\mathrm{SiC}$ crystal with both its lateral dimensions equal to one crystallographic period length $(=0.31 \times 0.53 \mathrm{~nm})$ and 12 layers of atoms $(=2.94 \mathrm{~nm})$ with a $1.75 \mathrm{~nm}$ vacuum layer along the vertical dimension. Electronic structure calculations are performed using Kohn-Sham DFT approach with the Vienna ab initio simulation package (VASP) [26]. Core electrons of each atom are assumed fixed using an ultrasoft pseudo-potential model. The exchange and correlation interactions of the electrons are approximated with the generalized gradient approximation (GGA). The simulation cell is discretized with a plane wave basis set having a plane wave cutoff energy of $240 \mathrm{eV}$. A Brillouin zone integration scheme having an $8 \times 8 \times 1 \mathrm{k}$-point grid is employed. These parameters, the 
simulation cell height, and vacuum layer thickness are varied and tested to ensure energy convergence. The generalized stacking fault (GSF) energies $E_{G S F}$ are calculated as a function of normalized displacement $(x / \mathbf{b})$ by rigidly shifting the atomic layers in the top part of the cell against the fixed bottom layers. The simulation cell is subsequently relaxed perpendicular to the slip plane to attain the minimum energy configuration.

\section{Results}

\subsection{In situ TEM uniaxial compression test}

From the mechanical responses of uniaxially compressed $\mathrm{SiC}$ pillars oriented along $\langle 0001\rangle$ and along $45^{\circ}$ with respect to $\langle 0001\rangle$, referred to as $\Phi_{0}$ and $\Phi_{45}$ respectively, we show that plastic flow in $\mathrm{SiC}$ is due to slip on the basal planes. These findings are consistent with ab initio theoretical analysis, which suggests that plastic slip at room-temperature is energetically most favorable on the basal plane and will occur by full dislocation glide on the shuffle set. In our experiments, we measure yield strengths $\sigma_{y}$ as high as $14.3 \mathrm{GPa}$. Both MD and DFT calculations suggest that these values are significantly below what is required to nucleate dislocations. Based upon our experimental, MD simulation, and DFT calculation results, we conclude that sustained plastic flow in $\mathrm{SiC}$ crystals at room temperature is governed by dislocation glide.

Figs. 1(a-g) show representative bright-field TEM images (extracted from " $6 \mathrm{H}-\mathrm{SiC} \Phi_{45}$ pillar" Movie), a selected area electron diffraction (SAED) pattern, and stress-displacement data recorded during uniaxial compression of a $\sim 600 \mathrm{~nm}$ long, $\Phi_{45}$-oriented $6 \mathrm{H}-\mathrm{SiC}$ pillar with diameter $D=180 \mathrm{~nm}$. In this experiment, the pillar is compressed until a nominal displacement of $100 \mathrm{~nm}$ after which the load is released. During compression, the initial stress-displacement response is linear, characteristic of elastic deformation, up to a point beyond which the 
displacement increases non-linearly with applied stress indicative of plastic flow as shown in Fig. 1(g). The TEM images, e.g., Fig. 1(b), corresponding to this transition reveal the generation of dislocations from the point of contact between the pillar and the diamond punch. After this initial event, additional bursts of dislocations are observed in the specimen. These events, highlighted by arrows labeled 1 and 2 in Figs. 1(c, d, and g), correspond to abrupt changes in the stress-displacement behavior. Fig. 1(e) is an image of the pillar obtained after compression. Clearly, the pillar is intact, without any visible cracks, and is $\sim 10 \%$ shorter in length, all characteristic of plastic deformation. SAED pattern (Fig. 1(f)) acquired from the pillar indicates that its crystallinity and crystal structure are not affected by the compression process. All of these results are typical of six $\Phi_{45}$ pillars with $D$ between $170 \mathrm{~nm}$ and $280 \mathrm{~nm}$. Although the stressdisplacement responses of these pillars are qualitatively similar, the extent of plastic hardening, the elastic to plastic transition point (i.e. the yield point, $\sigma_{y}$ ), and the loading/unloading curve slopes vary from pillar to pillar due to differences in alignment of the pillars (see Fig. S2 in Supplementary Information). We measure $\sigma_{y}$ values between $\sim 7.8$ and $14.3 \mathrm{GPa}$ for the different pillars, but $\sigma_{y}$ did not vary systematically with $D$ in our experiments.

In comparison, $\Phi_{0}$-pillars, i.e. $6 \mathrm{H}-\mathrm{SiC}(0001)$ pillars compressed uniaxially along $\langle 0001\rangle$, do not undergo plastic deformation but fail catastrophically via brittle fracture (see " $6 \mathrm{H}-\mathrm{SiC} \Phi_{0}$ pillar" Movie and Fig. S3 in Supplementary Information). In these $\Phi_{0}$-pillars, the displacement increases linearly with applied stress up to the point of failure, which occurs at stresses between 15 and $22 \mathrm{GPa}$. The lack of any contrast changes in the TEM images acquired during the test suggest that dislocation motion is either limited or absent in $\Phi_{0}$ pillars, as expected for the brittle fracture. We observe similar mechanical response in all the six $\Phi_{0}$ pillars with $D$ between 180 $\mathrm{nm}$ to $300 \mathrm{~nm}$. We attribute the ductility (brittleness) of $\Phi_{45}\left(\Phi_{0}\right)$ pillars to the activation 
(suppression) of slip on the basal planes at room-temperature and to much higher critical resolved shear stresses required to activate non-basal prismatic or pyramidal slip planes.

We note that the unloading portion of the stress-displacement curve in Fig. 1(g) is nonlinear. Such a deviation from linearity in the unloading curve, referred to as knee or elbow, has been observed during indentation of silicon and attributed to reversible pressure-induced phase transformation [27-29].

In order to check for any such phase transformations occurring within the $6 \mathrm{H}-\mathrm{SiC}$ pillars, we carried out detailed TEM characterization of the deformed pillars. Fig. 2(a) is a lowmagnification TEM image obtained after further thinning of the compressed pillar shown in Fig. 1. In this image, several dislocation lines are visible near the bottom of the pillar and slip traces at the top of the pillar are highlighted with a circle. Fig. 2(b) is a higher-magnification scanning TEM (STEM) image obtained from the area around a slip trace within the circle. Figs. 2(c) and 2(d) are high-resolution STEM images from the areas outlined by blue and red lines above and below the slip trace, respectively. In both these images, we observe alternate zigzag symmetry, characteristic of $6 \mathrm{H}-\mathrm{SiC}[30,31]$. We did not find any stacking faults or evidence of structural phase transformation in this deformed region, consistent with the assertion that the observed plastic flow is due to dislocation glide. During STEM characterization (see Fig. S4 in Supplementary Information for details) of this pillar, we found an interesting phenomenon: rotation of the crystal lattice near the slipped regions of the sample, a consequence of plastic flow during compression. Similar behavior has been observed in mechanically-deformed singlecrystalline metallic materials subjected to tensile loading [32].

In order to determine the mechanisms underlying the sustained plastic flow in the $6 \mathrm{H}-\mathrm{SiC}$ $\Phi_{45}$ pillars, we performed MD simulations of uniaxial compression and calculated the energies 
associated with slip using DFT. This combination of MD and DFT calculations enabled prediction of the most probable slip system and the corresponding critical stress.

\subsection{MD simulations}

Figures 3(a-d) are typical MD simulation images (see " $6 \mathrm{H}-\mathrm{SiC} \Phi_{45}$ pillar MD simulations" Movie for an animated version) obtained during uniaxial compression of a $6 \mathrm{H}-\mathrm{SiC}$ $\Phi_{45}$ pillar with $D=8.4 \mathrm{~nm}$. Fig. 3(e) is a plot of the stress as a function of strain obtained from the simulations. The pillar deforms elastically up to a strain of $\sim 8 \%$, corresponding to an applied stress of $\sim 20 \mathrm{GPa}$. At higher stress, the pillar exhibits non-uniform, elastic shear deformation, this behavior which occurred in the form of slip bands on the more easily-shearable atomic planes, depicted in Fig. 3(c) by higher von Mises strain, is followed by nucleation of a dislocation with full Burgers vector $\mathbf{b}=\langle 1120\rangle$ on the shuffle set of a $\{0001\}$ plane, consistent with the literature [33]. Our MD simulations predict that the critical compressive stress required

for dislocation nucleation is $31 \mathrm{GPa}$. Following nucleation, the dislocation eventually glides to the surface and forms an atomic step as shown in Fig. 3(d). Based on these results, we suggest that $6 \mathrm{H}-\mathrm{SiC} \Phi_{45}$ pillars, subjected to uniaxial compression, can undergo plastic deformation via slip by dislocation nucleation and glide on the shuffle set of basal planes.

We realize that the accuracy of empirical potential used in our MD simulations can be limited. Therefore, we have also carried out electronic structure calculations with DFT to determine energetically the most favorable pathways for dislocation glide.

\subsection{DFT calculations}


While theoretical predictions of plastic deformation in covalent materials is still a developing research area, the resistance to dislocation glide is often strongly correlated with the maxima in the generalized stacking fault energy $E_{G S F}$ and the gradient $\left(\mathrm{d} E_{G S F} / \mathrm{d} x\right)$ of $E_{G S F} v s$. rigid displacement $(x)$ curves. For a given slip system, the maximum value in the $\mathrm{d} E_{G S F} / \mathrm{d} x v s$. $(x / \mathbf{b})$ curve is the ideal shear stress $\left(\tau_{0}\right)$ required to destabilize a perfect crystal when sheared on that particular slip plane and slip direction. $\tau_{\mathrm{o}}$ is a measure of the critical stress at which a dislocation nucleates from a free surface in a pure crystal [34].

We calculated $E_{G S F} v s . x / \mathbf{b}$ for four different possible slip systems involving two sets of basal planes, the shuffle and the glide sets, and two slip directions, $\langle 1120\rangle$ and $\langle 1100\rangle$ using a crystal with periodic boundary conditions in the in-plane directions and the basal planes as the top and bottom surfaces. Figs. 4(a-d) show schematics of the simulation cells used. The calculated $E_{G S F}$ and $\mathrm{d} E_{G S F} / \mathrm{d} x$ data are plotted $v s .(x / \mathbf{b})$ in Figs. 4(e) and 4(f), respectively. For all the four slip systems, the $E_{G S F}$ increases monotonically with $x$ and the maxima is obtained at $x=$ $\mathbf{b} / 2$, and the maxima in $\mathrm{d} E_{G S F} / \mathrm{d} x$ (i.e. $\tau_{\mathrm{o}}$ ) are observed at $x=\mathbf{b} / 4$. From the data, we find that energetically the most favorable (lowest $\tau_{\mathrm{o}}$ ) and unfavorable (highest $\tau_{\mathrm{o}}$ ) slip systems are the basal slip on the shuffle set along $\langle 1100\rangle$ (the dashed curves in Figs. 4(e) and 4(f)) and basal slip on the glide set along $\langle 1120\rangle$ (the data is off-scale on both Figs. 4(e) and 4(f)), respectively. While the basal slip on the shuffle set along $\langle 1100\rangle$ consists of two distinct directions, with shearing first along $1 / 3[1 \overline{1} 00]$ and then along $1 / 3[10 \overline{1} 0]$, the lack of an intermediate energy minimum along this path suggests that the dislocation does not dissociate into partials separated by a stacking fault. The second most favorable slip path, i.e. the path with the second lowest $\tau_{0}$, is on the shuffle set along $\langle 1120\rangle$ corresponding to the solid curve in Fig. 4(f). The third possible 
basal slip is on the glide set along $\langle 1100\rangle$ that involves dissociation of the dislocation into two partials along $1 / 3[1 \overline{1} 00]+1 / 3[10 \overline{1} 0]$ separated by a stable stacking fault. This slip mechanism is considered to be active at high temperatures [35,36]. Although $E_{G S F}$ is the lowest for this process, shown by the dotted curve in Fig. 4(e), $\tau_{\mathrm{o}}$ is higher than those required for basal slip on the shuffle sets along $\langle 1100\rangle$ and $\langle 1120\rangle$ because the magnitude of the Burgers vector, $|\mathbf{b}|=$ $1 / \sqrt{6}$, is shorter compared to both $2 / \sqrt{6}$ along $\langle 1100\rangle$ and $1 / \sqrt{2}$ along $\langle 1120\rangle$. Based upon our calculations, we conclude that the plastic deformation of single-crystalline, defect-free, $6 \mathrm{H}$ $\mathrm{SiC} \Phi_{45}$ pillars occurs via glide of full dislocations on the shuffle set of the basal plane at roomtemperature. Our TEM analysis (see Fig. S5 in Supplementary Information) suggests that dislocations with $\mathbf{b}=[11 \overline{2} 0]$ are present in the deformed sample. While our results are qualitatively consistent with TEM observations of post-indented microstructures of $4 \mathrm{H}$ - and $3 \mathrm{C}$ SiC samples [33], we cannot rule out the possibility of operation of other pathways because the sample geometry and in situ TEM experimental set up has limited our ability to detect other dislocations. Additional detailed TEM characterization is necessary to help better understand the observed mechanical behavior.

We now focus on whether the experimentally-observed plastic flow in $6 \mathrm{H}-\mathrm{SiC}$ is controlled by nucleation or glide of dislocations. Under compression, the dislocations are likely to nucleate at the surface of pillars in contact with the diamond tip. However, due to the limited resolution of the in situ TEM, the onset of nucleation and the exact nucleation sites are difficult to determine in our experiments. Our DFT calculations of $\mathrm{d} E_{G S F} / \mathrm{d} x v s$. $(x / \mathbf{b})($ Fig. $4(\mathrm{f}))$ predict that the ideal shear stress (the lowest $\tau_{\mathrm{o}}$ ) associated with nucleation of a dislocation on the shuffle set along $\langle 1100\rangle$ of the basal plane surface is $\sim 33 \mathrm{GPa}$. The corresponding uniaxial compressive 
stress is $~ 66 \mathrm{GPa}$ (since Schmid factor is $1 / 2$ for this slip system) and is more than twice the stresses obtained from MD simulations. ${ }^{1}$ Nevertheless, both MD and DFT calculated stresses for dislocation nucleation are up to an order of magnitude higher than the experimentally determined $\sigma_{y}$. Therefore, we suggest that the observed plastic deformation in $6 \mathrm{H}-\mathrm{SiC}$ is due to existing dislocations rather than the nucleation of new dislocations. This assertion is consistent with the stresses expected for dislocation glide at room temperature in $6 \mathrm{H}-\mathrm{SiC}$ [37].

\section{Conclusions}

In summary, we have directly observed sustained plastic flow in $6 \mathrm{H}-\mathrm{SiC}$ pillars at room temperature. Using transmission electron microscopy along with ab initio modeling, we determined that the glide of full dislocations on shuffle set of basal planes leads to the observed plastic deformation. Based on our electronic structure calculations, we identify dislocation propagation along $1 / 3[1 \overline{1} 00]+1 / 3[10 \overline{1} 0]$ as energetically the most favorable path on the shuffle set of the basal plane. Motion of dislocations in compounds require multiple diffusional events and hence is often not expected to occur at room-temperature, especially in refractory carbides such as $\mathrm{SiC}$. Our results show that this phenomenon is indeed observable under high applied stresses, which in our experiments is found to be $7.8 \mathrm{GPa}$. In comparison, room-temperature plasticity has not been reported for bulk $6 \mathrm{H}-\mathrm{SiC}$ crystals, which are found to fracture at much lower stresses, $~ 3.6 \mathrm{GPa}$ [38]. The absence of plastic flow in bulk crystals is likely due to a higher density of structural defects compared to smaller crystals used in our experiments. It is plausible that carefully-prepared bulk $6 \mathrm{H}-\mathrm{SiC}$ crystals with relatively low density of defects are

\footnotetext{
1 The discrepancy between the MD and DFT predicted values can be attributed to (1) surface roughness of the MD crystal, (2) limited accuracy of the MD empirical potential, and/or (3) small cell sizes and rigid displacement constraints inherent to DFT calculations.
} 
likely to exhibit room-temperature plasticity. Therefore, future studies focused on relating the observed mechanical responses of $\mathrm{SiC}$ to the nature and density of dislocations within the bulk and small-scale crystals are desirable. Our results open up an exciting possibility of designing nanomechanical and nanoelectromechanical systems with hard-yet-ductile $\mathrm{SiC}$ and possibly other ceramic components with potentially enhanced performances.

\section{Acknowledgments}

We gratefully acknowledge support from the AFOSR (Dr. Ali Sayir) FA9550-10-1-0496 and FA9550-11-10273. The in situ electron microscopy experiments were conducted as part of a user project at the National Center for Electron Microscopy, Lawrence Berkeley National Laboratory, supported by the Office of Science, Office of Basic Energy Sciences, of the U.S. Department of Energy under Contract No. DE-AC02-05CH11231. We thank Marissa Manusco for her help with the sample preparation. VR acknowledges support from Nanotechnology and Functional Material Center, and the Ministry of Education and Science of the Republic of Serbia, project \# 172054. 


\section{References}

[1] Uchic MD, Dimiduk DM, Florando JN, Nix WD. Science 2004;305:986.

[2] Greer JR. Rev Adv Mater Sci 2006;13:59.

[3] Fujita S, Maeda K, Hyodo S. J Mater Sci Lett 1986;5:450.

[4] Fujita S, Maeda K, Hyodo S. Philos Mag A: Phys Condens Matter, Struct Defect Mech Prop 1987;55:203.

[5] Maeda K, Suzuki K, Fujita S, Ichihara M, Hyodo S. Philos Mag A: Phys Condens Matter, Struct Defect Mech Prop 1988;57:573.

[6] Samant A, Pirouz P. Int J Refract Met Hard Mater 1998;16:277.

[7] Corman GS. J Am Ceram Soc 1992;75:3421.

[8] Mussi A, Rabier J, Thilly L, Demenet JL. Phy Stat Sol 2007;4:2929.

[9] Lancin M, Texier M, Regula G, Pichaud B. Philos Mag 2009;89:1251.

[10] Sawyer GR, Sargent PM, Page TF. J Mater Sci 1980;15:1001.

[11] Eddy CR, Wu P, Zwieback I, VanMil BL, Myers-Ward RL, Tedesco JL, Souzis AE, Gaskill DK. Mater Sci Forum, Trans Tech Publ 2009; 615:323.

[12] Shim S, Jang JI, Pharr GM. Acta Mater 2008;56:3824.

[13] Wong EW, Sheehan PE, Lieber CM. Science 1997;277:1971.

[14] Cheng G, Chang TH, Qin Q, Huang H, Zhu Y. Nano Lett 2014;14:754.

[15] Han XD, Zhang YF, Zheng K, Zhang XN, Zhang Z, Hao Y, Guo X, Yuan J, Wang Z. Nano Lett 2007:7:452.

[16] Y. Zhang, Han X, Zheng K, Zhang Z, Zhang X, Fu J, Ji Y, Hao Y, Guo X, Wang ZL. Adv Funct Mater 2007;17:3435.

[17] Snead LL, Nozawa T, Katoh Y, Byun TS, Kondo S, Petti DA. Adv Funct Mater 2007;371:329.

[18] Yin L, Vancoille EY, Ramesh K, Huang H. Int J Mach Tool Manufact 2004;44:607.

[19] Robert SO. The MEMS Handbook, Florida: CRC Press; 2001.

[20] Plimpton S. J Comput Phys 1995;117:1.

[21] Devanathan R, Diaz de la Rubia T, Weber WJ. J Nucl Mater 1998;253:47.

[22] Schneider A, Kaufmann D, Clark B, Frick C, Gruber P, Mönig R, Kraft O, Arzt E. Phys Rev Lett 2009;103:105501.

[23] Verlet L. Phys Rev 1967;159:98.

[24] Clausius R. Philos Mag 1870;40:122.

[25] Li J. Model Simul Mater Sci Eng 2003;11:173.

[26] Kresse G, Furthmüller J. Comput Mater Sci 1996;6:15.

[27] Rao R, Bradby JE, Ruffell S, Williams JS. Microelectron J 2007;38:722.

[28] Juliano T, Gogotsi Y, Domnich V. J Mater Res 2003;18:1192.

[29] Gerbig YB, Stranick SJ, Morris DJ, Vaudin MD, Cook RF. J Mater Res 2009;24:1172.

[30] Jepps NW, Page TF. Prog Cryst Growth Charact Mater 1983;7:259.

[31] Pirouz P, Yang JW. Ultramicroscopy 1993;51:189.

[32] Courtney TH. Mechanical Behavior of Materials, New York: McGraw Hill; 2000.

[33] Demenet JL, Amer M, Tromas C, Eyidi D, Rabier J. Phys Status Solidi C 2013;10:64.

[34] Umeno Y, Kinoshita Y, Kitamura T. Model Simul Mater Sci Eng 2007;15:27.

[35] Samant AV, Hong MH, Pirouz P. Phys Status Solidi B 2000;222:75.

[36] Ha S, Benamara M, Skowronski M, Lendenmann H. Appl Phys Lett 2003;83:4957.

[37] Samant AV, Zhou WL, Pirouz P. Phys Status Solidi A 1998;166:155. 
[38] Wachtman JB. Structural Ceramics, Elsevier; 2012. 


\section{List of Figures}

Fig. 1. (a-d) Bright-field TEM images acquired in situ during displacement-controlled uniaxial compression of a single-crystalline $6 \mathrm{H}-\mathrm{SiC}$ pillar oriented at $45^{\circ}$ with respect to $\langle 0001\rangle$, referred to as $\Phi_{45}$. The pillar diameter $D=180 \mathrm{~nm}$. In this experiment, the applied load is increased until a nominal displacement of $100 \mathrm{~nm}$ and released. e) Image of the pillar after compression. f)

Selected area electron diffraction (SAED) pattern obtained along [11 $\overline{2} 0]$ zone axis. g) Plot of engineering stress $v s$. displacement data obtained during the compression test. Arrows 1 and 2 highlight displacement bursts corresponding to slip on $\{0001\}$ planes.

Fig. 2. (a) Typical bright-field TEM image of the compressed pillar shown in Fig. 1 obtained after further thinning by focused ion beam milling. (b) Higher-magnification scanning TEM (STEM) image of the region around the slip trace highlighted by a circle in Fig. 2(a). Highresolution STEM images of the regions (c) above and (d) below the slip trace, outlined by the blue and red lines, respectively. These images are obtained with zone axis along [1120]. (See Fig. S4 and associated description in the Supplementary Information for imaging details) 
Fig. 3. (a-d) Molecular dynamics (MD) simulation images obtained during uniaxial compression of a 24-nm-long $6 \mathrm{H}-\mathrm{SiC} \Phi_{45}$ pillar $(D=8.4 \mathrm{~nm})$ at $300 \mathrm{~K}$. Stress is applied by imposing deformation to the pillar. (e) Plot of compressive stress vs. strain calculated from the MD simulations. The images labeled (a)-(d) correspond to the points (a) to (d) in the plot and show: (a) uniform elastic deformation, (b) non-uniform shear deformation along slip bands, (c) dislocation nucleation on the shuffle set of the basal plane from the bottom surface, and (d) dislocation glide to the surface to form an atomic step. The bulk atoms are colored by von Mises strain, with blue and red colors corresponding to minimum and maximum strains, respectively.

Fig. 4. Schematics of the simulation cells used for computing generalized stacking fault energies $E_{G S F}$ for (top) shuffle and (bottom) glide sets of the basal plane shearing along (a,c) $\langle 1120\rangle$ and (b,d) $\langle 1100\rangle$. Dashed horizontal lines indicate locations of the slip planes in both the panels. The slipped images correspond to the unstable stacking fault configurations. Arrows highlight the slip paths taken to obtain the $E_{G S F}$ curves shown in Fig. $4(\mathrm{e}):\langle 1120\rangle / 2+\langle 1120\rangle / 2$ for (a and c), $[1 \overline{1} 00] / 3+[10 \overline{1} 0] / 3$ for (b), and $\langle 1100\rangle / 6+\langle 1100\rangle / 6$ for (d). Plots of (e) $E_{G S F}$ and (f) $\mathrm{d} E_{G S F} / \mathrm{d} x$ vs. position $(x / \mathbf{b})$, where $\mathbf{b}$ is the Burgers vector. The solid, dashed, and dotted curves represent dislocation glide depicted in (a), (b), and (d) respectively. $E_{G S F}$ and $\mathrm{d} E_{G S F} / \mathrm{d} x$ values for path (c) are over $2 \times$ higher than those of the other paths and hence are not shown in these plots. 

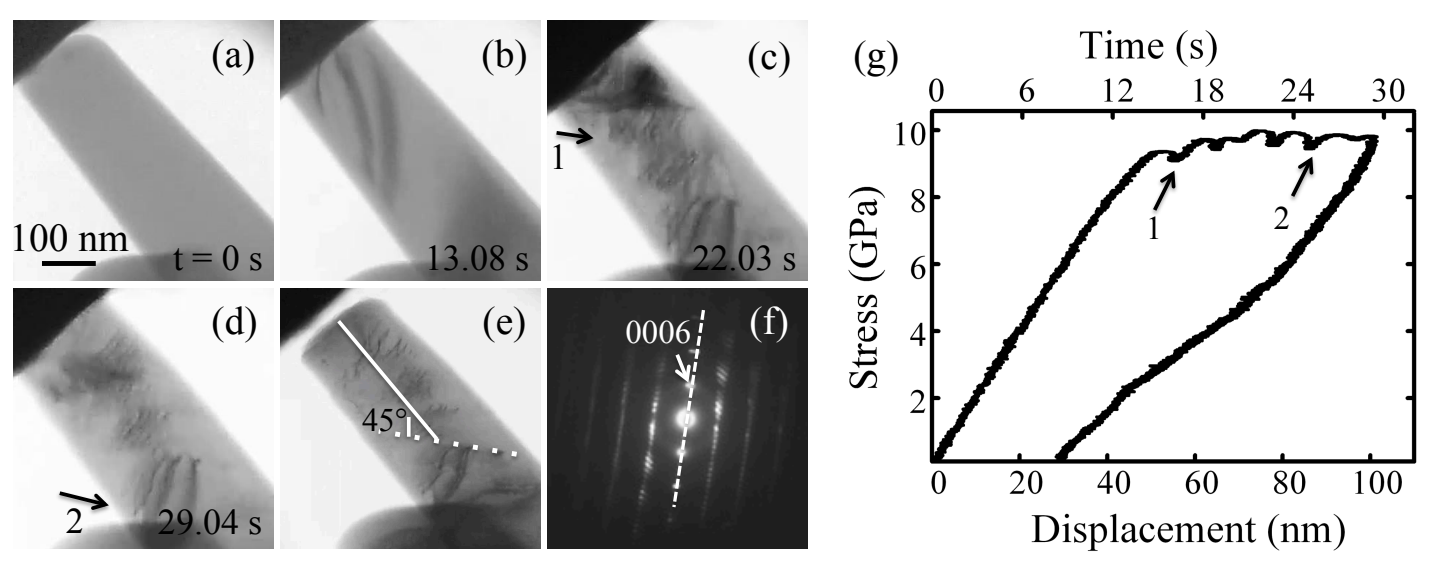


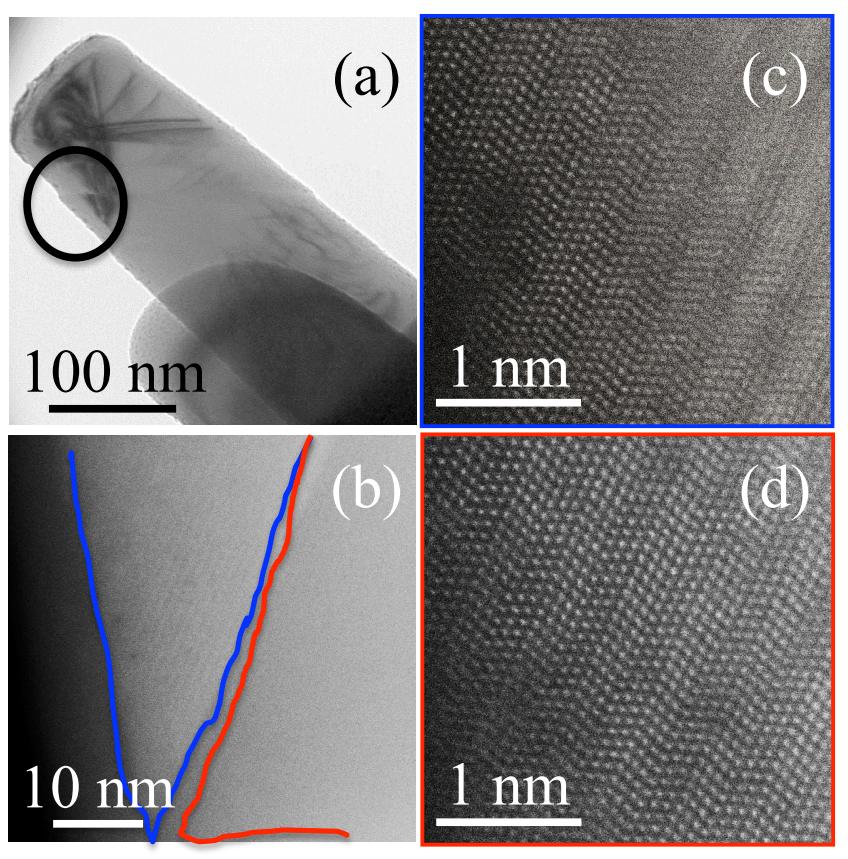



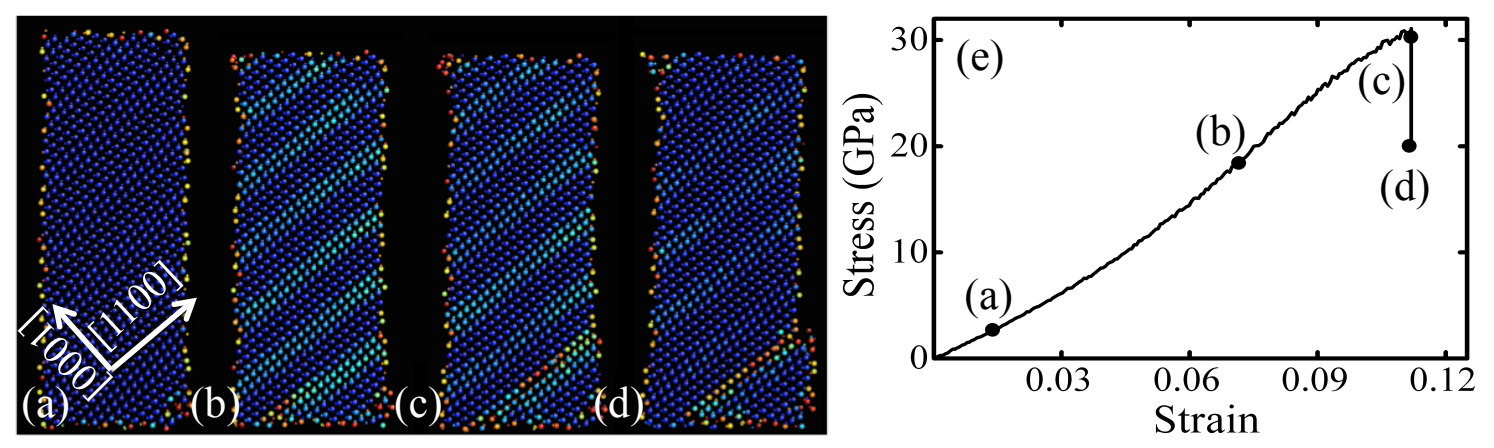


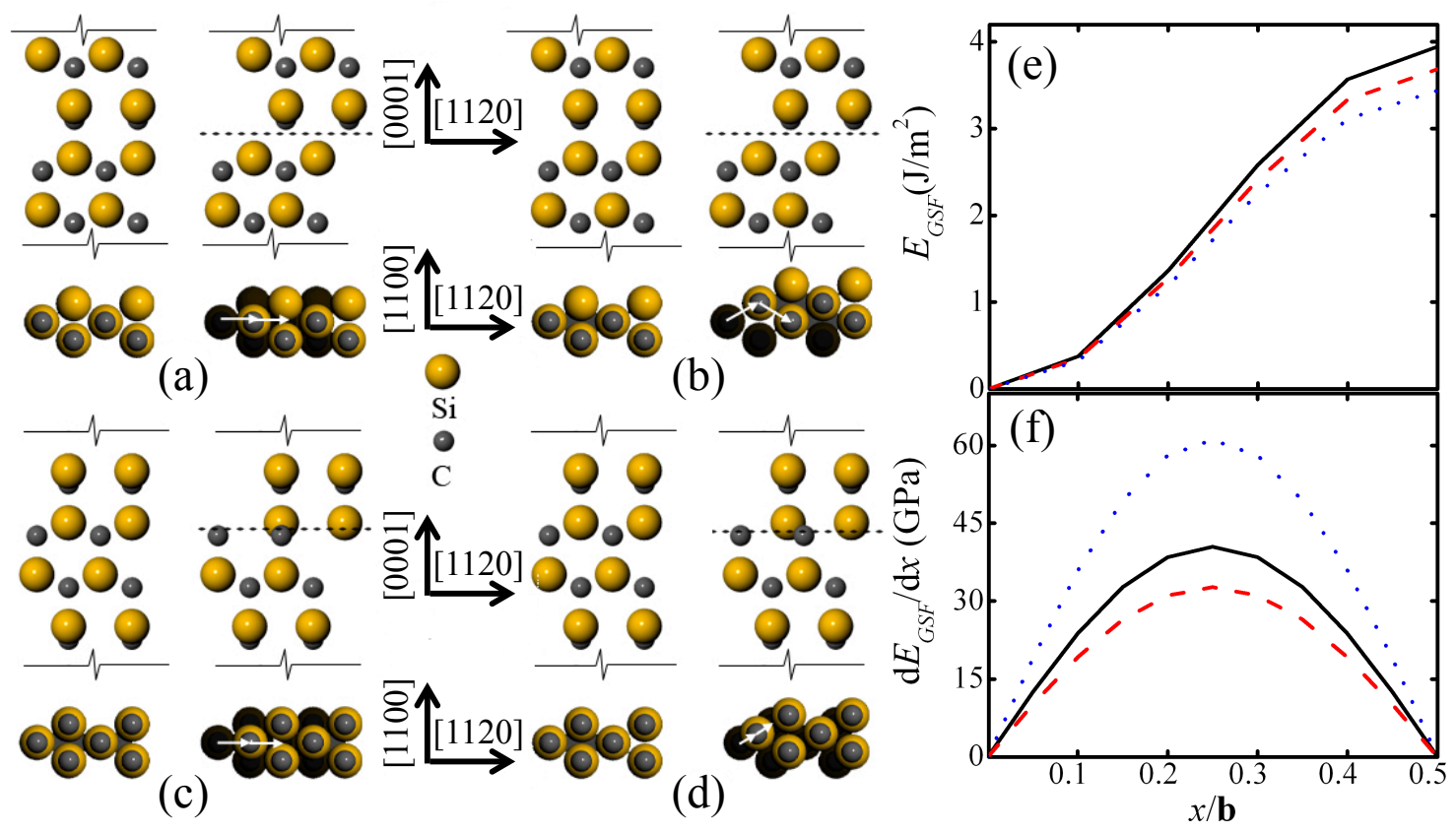

